\title{
Exploring the financial risk of a temperature index: a fractional integrated approach
}

\author{
Rosella Castellano $^{a}$, Roy Cerqueti $^{b}$, Giulia Rotundo $^{c}$ \\ ${ }^{a}$ University of Roma Unitelma-Sapienza, Department of Law and Econommics \\ V.le Regina Elena 295, 00161, Rome, Italy. \\ Tel.: +39 06-81100288; fax: +39 06-6792048. Email: rosella.castellano@unitelma.it. \\ ${ }^{b}$ University of Macerata, Department of Economics and Law. \\ Via Crescimbeni 20, 62100, Macerata, Italy. \\ Tel.: +390733 2583246; fax: +390733 2583205. Email: roy.cerqueti@unimc.it. \\ ${ }^{c}$ Sapienza University of Rome \\ Department of Statistical Sciences, \\ p.le A. Moro 5, 00185, Rome, Italy. \\ Tel.: +39 064976 6404; fax +390649766765. Email: giulia.rotundo@uniroma1.it.
}

July 24, 2018

\begin{abstract}
This paper introduces a new temperature index, which can suitably represent the underlying of a weather derivative. Such an index is defined as the weighted mean of daily average temperatures measured in different locations. It may be used to hedge volumetric risk, that is the effect of unexpected fluctuations in the demand/supply for some specific commodities - of agricultural or energy type, for example due to unfavorable temperature conditions.

We aim at exploring the long term memory property of the volatility of such an index, in order to assess whether there exist some long-run paths and regularities in its riskiness. The theoretical part of the paper proceeds in a stepwise form: first, the daily average temperatures are modeled through autoregressive dynamics with seasonality in mean and volatility; second, the assessment of the distributional hypotheses on the parameters of the model is carried out for analyzing the long term memory property of the volatility of the index. The theoretical results suggest that the single terms of the index drive the long memory of the overall aggregation; moreover, interestingly, the proper selection of the parameters of the model might lead both to cases of persistence and antipersistence. The applied part of the paper provides some insights on the behaviour of the volatility of the proposed index, which is built starting from single daily average temperature time series.
\end{abstract}




\section{Introduction}

Weather derivatives represent a particular kind of exotic financial contract introduced to manage the volumetric risk caused by unfavorable weather conditions. Since their inception, they have represented an alternative to standard insurance contracts which mainly offer protections against extreme weather events. Weather derivatives instead can also be used to hedge other types of risks, including the usual uncertainty of weather conditions, which are more likely to occur.

The main difference between weather derivatives and traditional ones is that the underlying of the first ones is a non-tradable weather index (i.e. rainfall, temperature, humidity or snowfall, or any other weather variable), meaning that their primary function is to hedge the volume risk resulting from changes in the supply/demand for goods due to adverse and unexpected weather changes (Muller and Grandi, 2000; Geman, 2005), rather than the price risk. Indeed, price risk can be hedged more effectively by means of standard commodity derivatives.

In view of the considerable portion of economy vulnerable to weather, of the increased number of concluded deals and the widened range of products offered, weather derivatives play an important role in integrated risk management and diversification, especially in the North American markets (Buckley et al., 2002).

Moreover, the last years have shown an expansion of studies on the usage of temperatures for the hedging of natural phenomena. Probability distributions and hypothesis testing are used for the development of models. For instance, in (Bloch et al., 2011) - by implementing a statistical analysis of the temperature time series - an Ornstein-Uhlenbeck process is considered for the dynamics of the global mean temperature. Such a model is then used as basis for a realistic new semi-empirical model for estimating the global sea-level response.

There is also evidence of the employment of weather derivatives by companies to implement business strategies for covering excess costs and reducing the volatility of revenues (see e.g. Zara, 2010; Golden et al., 2007; Yang et al., 2011). Moreover, Yang et al. (2011) focuses also on the role of weather derivatives for diversifying portfolio investments due to the low correlation of such products with standard financial stocks.

Thus, the exploration of weather derivatives risk is of paramount relevance. In this respect, we also mention the nice paper of Stulek (2017), which discusses how weather derivatives risk management could be seen as a suitable device for mitigating risk in retail. The authors present a special focus on the case of Croatian food stores.

At the moment, weather derivatives are usually structured as swaps, futures and options written on different weather indexes such as Daily Average Temperature (DAT), Cumulative Annual Temperature (CAT), Heating 
Degree Days (HDDs), Cooling Degree Days (CDDs), precipitation, snowfall and wind. The underlying can be a single weather variable or a combination of them.

In this work, we analyse the second type of underlying. In particular, we consider a weather index which is defined as a linear combination of several DATs, detected in different geographic areas. The volatility of the index is given by the weighted mean of the volatilities of DATs. In so doing, we are in line with the evidence suggesting that a weather derivative written on a temperature index, i.e. a set of DATs, might contribute to hedge the volumetric risk associated with a particular product, in a specific geographic area (see e.g. Gülpinar and Canakoglu, 2017). Furthermore, in the proposed model a dependence structure for the DATs is introduced, according to a wide part of the literature (see e.g. Alexandridis and Zapranis, 2013; Benth and Saltyte Benth, 2013 and Saltyte Benth et al., 2007).

In particular, we focus on the analysis of a relevant aspect of the risk associated with the index, that is its long term memory (or persistence) property. Adopting the perspective of a risk-driven agent, whose financial decisions are assumed to be remarkably affected by the volatility of the underlying, the risk analysis of the underlying index might provide useful insights on the profile of the derivative itself. Moreover, the long term memory provides a key information on how a system reacts to shocks - an issue of paramount relevance in a very volatile context, as weather.

In analyzing the long term memory of the temperature volatility, we follow the route traced by empirical studies on climate and geophysical science, which state that the series of the temperature exhibits persistence properties (see e.g. Syroka and Toumi, 2001; Tsonis et al., 1999).

Moreover, it is worth recalling that long memory is strictly interconnected to the so-called Joseph effect (see the breakthrough paper by Mandelbrot and Wallis, 1968 but also Eliazar and Klafter, 2005). For what concerns specifically weather derivatives, the matter is that in an increasingly interconnected world, the long term memory of the aggregate (weather indices) may help in driving the overall super-national productions, distribution, foresee the need of good and services, alongside estimate of the occurrence of extreme events and readiness of response. In this respect, we notice that the estimate of the underlying is a key point for the estimate of the related derivative.

In general, the informative content of temperature long term memory in terms of financial modelling is clear. It is worth mentioning Brody et al. (2002) and Benth (2003) who introduce a Ornstein-Uhlenbeck stochastic process driven by a Fractional Brownian Motion for the daily-mean temperature evolution, in the context of weather derivatives. In so doing, the long term property of the considered series is taken into account.

The long term memory represents an important property of time series - even those generated by stochastic processes - and is associated with 
the hyperbolic decay of the autocorrelation function with respect to time lag. Thus, the long term memory can provide information on how strongly systems depend on past realizations and, consequently, how fast they recover from good or bad shocks.

In the framework of risk models, the long memory of prices volatility might be employed for explaining the deviations from linear models for performing forecast on future prices (see e.g. Bouchaud et al., 2004; Lillo et al., 2005). Thus, our model contributes by one side to the challenging theme of future weather derivatives price forecast and, by the other side, to the field of weather dynamics macrostructural statistical properties.

The earliest studies on long term memory appeared in the context of hydrogeological data, and are due to Hurst (1951 and 1957), Mandelbrot and Wallis (1968), McLeod and Hipel (1978), Smith and Harris (1987), among others.

In this framework, also financial markets and financial time series have been explored. The persistence property has been shown to be one of the features of many financial time series: speculative returns (Bollerslev and Mikkelsen, 1996; Ding and Granger, 1996), foreign exchange rate returns along with their power transformations (Ivanova and Ausloos, 1999; Ausloos and Ivanova, 2000; Reboredo et al. 2013; Ding and Granger, 1996), stock prices (Vandewalle and Ausloos, 1998; Ausloos and Ivanova, 1999; Reboredo et al. 2013). In the specific case of commodity prices, it is worth to mention the contributions of Wei and Leuthold (2000) on agricultural futures, Zhao et al. (2015) on oil prices and Cheung and Lai (1993) and Lo (1991) on gold market returns.

In this paper, we focus on a theoretical approach for assessing the long term memory property of a temperature index. To this aim, we analyze the stochastic process describing the riskiness of such an index. In particular, we explore the probabilistic requirements that must be satisfied by the parameters of the considered process for deriving the presence of the long term memory property.

The approach we follow is radically different from the one used by most of the papers in this field since, commonly, the persistence property of a time series is assessed through numerical procedures (see e.g. Bianchi et al., 2013, Lux and Ausloos, 2002, Markovich and Kilpi, 2009).

We advance a theoretical proposal, bringing this paper out of the framework of the nonparametric empirical analysis. Specifically, we are able to check long term memory for series described by time-dependent evolutive models without the need of collecting data.

The temperature index proposed here is a weighted average of DATs. Therefore, we analyze the long term memory of its volatility similarly to an agent-based model. This approach is particularly suitable to describe different sources of heterogeneity among interacting elements and, hence, to capture the heterogeneity of the different paths of temperatures composing 
the averaged weighted index, so that here the role of agents is played by temperatures.

Some examples of agent-based models in financial markets can be found in: Brock and Hommes $(1997,1998)$ and Hommes (2001). It is also important to mention Bunn and Oliveira (2003), and Zambrano and Olaya (2016) which - as we do here - deal with an agent-based model for commodity markets through simulations. By a different perspective, the presence of several units capturing different geographical locations of temperature measurements points to spatial evolutive models for weather derivatives (see e.g. Saltyte Benth et al., 2007; Hardle and Osipenko, 2011 and Saltyte Benth and Saltyte, 2011).

The usefulness of a theoretical assessment of the long-term memory of time series is twofold: first, the theoretical approach allows to overcome computational complexity related with numerical procedures and consequent estimation errors; second, the mathematical estimation of the Hurst exponent leads to undisputable results, not affected by parameters selections and characteristics of the considered sample.

In this work, we have been inspired by some contributions on the theoretical estimation of the Hurst exponent generated by stochastic processes (see e.g. Foellmer, 2005; Kirman and Teyssiere, 2005; Cerqueti and Rotundo, 2012; Zaffaroni, 2007, and the review proposed by Cerqueti and Rotundo, 2015). According to the above mentioned contributions, we discuss the distributional hypotheses of the parameters of a temperature index to verify the presence of long term memory in its volatility. Since such an index is a weighted aggregation of univariate DATs, we apply a very important result due to Granger (1980). It allows to derive the persistence properties of an index by the knowledge of the ones associated with its single components.

The applied part of the paper proceeds in a stepwise form. First, via a time series approach, we focus on the DAT dynamics of four cities located in the Eastern area of US: Baltimore, Boston, Cincinnati and Philadelphia. To capture seasonality, truncated Fourier series have been included in both mean and volatility. In the attempt of incorporating the stylized facts of temperature data, autoregressive models for conditional variance have been also considered. The proposed time series approach allows to take into account the noticeably differences in seasonal fluctuations observed among cities, both in terms of amplitude and frequency. Secondly, we build the temperature index and estimate the Hurst coefficient of its conditional volatility, which is obtained as weighted average of the conditional volatility of each DAT time series.

The paper is structured as follows. Section 2 discusses the model for daily average temperatures and defines the index which can be considered as the underlying of a weather derivative. Section 3 presents the theoretical approach for assessing the long term memory property of the volatility of the temperature index. Section 4 analyzes the theoretical result. Section 5 
presents an analysis based on observed data and Section 6 concludes.

\subsection{Daily Average Temperature: a brief review of literature}

DATs dynamics are characterized by predictable trends and seasonality, especially over longer horizons. This implies that temperatures are approximately predictable in the short run and random around historical averages in the long run. From the perspective of DATs modeling, it is possible to identify several approaches (we refer to Shiller et al., 2012, for a review). One of the possible alternatives is represented by the Index Modelling approach, focusing on the distribution of weather indices. A few papers suggest that this approach yields stable estimations if the distribution can be estimated relatively well (Dorfleitner and Wimmer, 2010). Geman and Leonardi (2005) study the statistical properties of both HDD and Accumulate HDD indices, and conclude that modeling directly the HDDs is not appropriate. Davis (2001) models the Accumulate HDD index by using a log-normal diffusion and concludes that this approach affects the estimates by $\pm 10 \%$.

Under a different approach, one can focus on dynamic models for DATs, so that the estimated models can be used to derive the indices. This second approach is the one used in this paper.

In principle, using DAT models can lead to a more accurate estimation than directly modelling temperature indices, since they make a complete use of available historical data. Moreover, when using index modeling, a different process must be estimated for each index. However, it is worth to mention that deriving accurate models for DATs is not straightforward since observed data show seasonality and evidence of long memory in the autocorrelation, so that small misspecifications of the model can lead, for instance, to large errors in the pricing of temperature-based weather derivatives (Alexandridis and Zapranis, 2013).

In literature, several processes have been proposed for modeling DATs. Moreno (2000) uses discrete-time mean reverting processes in which the volatility is adjusted via an arbitrarily-chosen sinusoidal function. This allows to take into account the seasonal component of the volatility, i.e. the volatility of temperature during summer is assumed to be different from that during winter. To capture the unique characteristics of DATs, Cao and Wei (2004) and Cao et al.(2004) use a discrete process which is built on five assumptions on DATs: follow a predicted cycle; move around a seasonal mean; are affected by global warming and urban effects; appear to have autoregressive changes and their volatility is higher in winter than in summer. Franses et al. (2001) consider a GARCH model (Engle, 1982; Bollerslev, 1986). Campbell and Diebold (2005) extend the ideas proposed by Cao and Wei (2004) and Franses et al. (2001), and consider a model for DATs in which the conditional mean dynamics include a trend, together with seasonal and cyclical components. They use a simple low-ordered polynomial determin- 
istic trend, while cyclical dynamics are captured by using autoregressive lags. To model seasonality, the Authors introduce a low-ordered Fourier series whose benefits are twofold. It produces a smooth seasonal pattern, which accords with the basic intuition that the progression through different seasons is gradual rather than discontinuous, and promotes parsimony, which enhances numerical stability in the estimates. Campbell and Diebold (2005) also consider conditional variance dynamics, with contributions coming from both cyclical and seasonal components. The seasonal component of volatility is approximated by a Fourier series, while the cyclical one is approximated by a non linear GARCH process. The idea of modelling DATs' volatility using GARCH is retaken also by Taylor and Buizza $(2004,2006)$.

\section{The model}

In this paper we move from the contributions mentioned in the previous Section. To model each single DAT composing the temperature index, we introduce a stochastic process assumed to be defined in a filtered probability space $\left(\Omega, \mathcal{F}, \mathbb{F}=\left(\mathcal{F}_{t}\right)_{t}, P\right)$.

The mean dynamics are calibrated by using $P$ autoregressive lags. Each DAT, $T_{i}$, under the assumption of normality in the residuals, is formalized as follows:

$$
T_{i}(t)=F_{i, t}+\sum_{p=1}^{P} \phi_{i, p} T_{i, t-p}+\varepsilon_{i, t} z_{t}
$$

where $\left(\varepsilon_{i, t}\right)_{t}$ is an i.i.d. process with finite mean and variance $\sigma_{\varepsilon}^{2},\left(z_{t}\right)_{t}$ is independent from $\left(\varepsilon_{i, t}\right)_{t}$ and such that $z_{t} \sim N(0,1)$, for each $t ;\left(\phi_{i, p}\right)_{i, p}$ is a $P \times N$ matrix of real numbers and $\left(F_{i, t}\right)_{i, t}$ is a deterministic seasonality function, which can be written as a truncated Fourier series as follows:

$$
F_{i, t}=\mu_{i}+\sum_{l=1}^{L_{i}}\left(a_{i, l} \cos \left(2 \pi l \frac{d_{i}(t)}{365}\right)+b_{i, l} \sin \left(2 \pi l \frac{d_{i}(t)}{365}\right)\right), \quad \forall i, t
$$

where $L_{i} \in \mathbb{N}$ is a truncation parameter, for each $i=1, \ldots, N$, the $a$ 's and the $b$ 's are real numbers and the $d$ 's are deterministic functions.

Notice that the presence of the common factor $z_{t}$ guarantees the existence of a dependence structure among the DATs.

We highlight here that the assumed distribution for the DAT's in formula (1) is rather general and is in line with the contributions mentioned in Subsection 1.1. Specifically, due to empirical evidence, we assume the presence of a seasonality term for each temperature series. Such a term is 
captured by a Fourier series, which is the standard mathematical device used for describing waves and seasonality. Moreover, we consider an autoregressive discrete-time model, which is widely employed in this context. Finally, we reasonably assume that DAT's are mutually dependent, as the common factor term suggests.

It is worth to recall here that DATs shows some kind of seasonality since variance turns out to be higher during winter times (Cao and Wei, 2004; Campbell and Diebold, 2005). For this reason, we model the conditional variance via a low-ordered Fourier series. Moreover, DATs exhibit some of the stylized facts common to financial asset returns, i.e. volatility clustering and fat tails.

Therefore, we assume that:

$$
\varepsilon_{i, t}^{2}=s_{i, t}+\beta_{i}+\eta_{i} \varepsilon_{i, t-1}^{2}+S_{i, t}+\gamma_{t},
$$

where:

$$
s_{i, t}=\sum_{j=1}^{J_{i}}\left(c_{i, j} \cos \left(2 \pi j \frac{d_{i}(t)}{365}\right)+f_{i, j} \sin \left(2 \pi j \frac{d_{i}(t)}{365}\right)\right)
$$

with a truncation parameter $J_{i} \in \mathbb{N}$, for each $i=1, \ldots, N$, real numbers $c$ 's and the $f$ 's and deterministic functions $d$ 's .

Furthermore, $\beta_{i} \in \mathbb{R}$ is the intercept, $\left(S_{i, t}\right)_{t}$ and $\left(\gamma_{t}\right)_{t}$ are i.i.d. stochastic processes with zero mean, representing the two sources of random noises - the former one of idiosyncratic nature, i.e. dependent on the specific temperature $T_{i}$; the latter of common nature, i.e. dependent only on time - while $\forall i, \eta_{i}$ is a random variable with support $(0,1)$ and represents the coefficient of the autoregressive term, for each $i=1, \ldots, N$.

The index we propose is a linear combination of the T's. Such a combination is selected in such a way that the risk of the new index is a weighted mean of the risk of the single components (see formula (5) for more details on this). We denote it, at time $t$, by $A(t)$, so that:

$$
A(t)=\sum_{i=1}^{N} w_{i} T_{i}(t)
$$

where $w_{i} \in[0,1]$ for each $i=1, \ldots, N$, and $\sum_{i=1}^{N} w_{i}^{2}=1$.

Remark 2.1. The proposed index is more general than the standard univariate ones, since the unidimensional case can be derived from the multidimensional one by taking all the weight w's equals to zero exept one.

Moreover, it is important to note that introducing a basket of DATs allows a 
high level of flexibility in the selection of the geographical area for which we want to measure the risk of unexpected changes in temperatures. Therefore, the proposed index allows an effective control of the volume risk level in the geographical area of interest.

The normalizing condition on the squared $w$ 's meets the requirement on the risk of the index, which is built as a weighted mean. In fact, the conditional variance of $A(t)$ given the information set at time $t-1$ is given by:

$$
\begin{gathered}
\operatorname{Var}\left(A(t) \mid \mathcal{F}_{t-1}\right)= \\
=\operatorname{Var}\left[\sum_{i=1}^{N} w_{i}\left(\sum_{p=1}^{P} \phi_{i, p} T_{i, t-p}+F_{i, t}+\varepsilon_{i, t} z_{t}\right) \mid \mathcal{F}_{t-1}\right]=\sum_{i=1}^{N} w_{i}^{2} \mathbb{E}\left(\varepsilon_{i, t}^{2} \mid \mathcal{F}_{t-1}\right),
\end{gathered}
$$

where $\mathbb{E}$ is the expected value operator.

Equation (5) states that the $N$ random variables $\varepsilon_{1, t}^{2}, \ldots, \varepsilon_{N, t}^{2}$ are quantities contributing in determining the volatility of index $A(t)$.

Moreover, rather than considering the $\varepsilon^{2}$ 's, we aim at analyzing the volatility of the index $A$ when the seasonality term is removed from the volatilities of each single DAT composing the index. Indeed, after eliminating the seasonality dependent variance from the residuals of each component, the autocorrelation of residuals became smaller and does not exhibit a clear seasonal pattern.

This means that we consider

$$
\tilde{\varepsilon}_{i, t}^{2}=\varepsilon_{i, t}^{2}-s_{i, t}, \quad \forall i, t .
$$

Specifically, we need to look at the random variable

$$
\mathbf{R}_{t}=\sum_{i=1}^{N} w_{i}^{2} \tilde{\varepsilon}_{i, t}^{2},
$$

whose expected value conditional to $\mathcal{F}_{t-1}$ is the volatility of $A(t)$. In fact, the volatility of the index remains unchanged when $\varepsilon_{i, t}^{2}$ is replaced by $\tilde{\varepsilon}_{i, t}^{2}$ as in (6), in that

$$
\mathbb{E}\left(\tilde{\varepsilon}_{i, t}^{2} \mid \mathcal{F}_{t-1}\right)=\mathbb{E}\left(\varepsilon_{i, t}^{2} \mid \mathcal{F}_{t-1}\right)
$$

\section{The assessment of long-term memory}

In order to theoretically derive the properties of long-term memory of each DAT, we now introduce the time-lag operator $L$, so that $L x_{t}=x_{t-1}$ for a 
generic stochastic process $\left(x_{t}\right)_{t}$. Equation (3) can be rewritten accordingly as follows:

$$
\left(1-\eta_{i} L\right) \tilde{\varepsilon}_{i, t}^{2}=\beta_{i}+S_{i, t}+\gamma_{t}
$$

hence

$$
\tilde{\varepsilon}_{i, t}^{2}=\frac{\beta_{i}}{1-\eta_{i} L}+\frac{S_{i, t}}{1-\eta_{i} L}+\frac{\gamma_{t}}{1-\eta_{i} L} .
$$

Thus,

$\mathbf{R}_{t}=\sum_{i=1}^{N} w_{i}^{2} \frac{\beta_{i}}{1-\eta_{i} L}+\sum_{i=1}^{N} w_{i}^{2} \frac{S_{i, t}}{1-\eta_{i} L}+\sum_{i=1}^{N} w_{i}^{2} \frac{\gamma_{t}}{1-\eta_{i} L}=: R_{t}^{(\beta)}+R_{t}^{(S)}+R_{t}^{(\gamma)}$

Each component of $\mathbf{R}_{t}$ in equation (10) has a precise meaning. In particular, $R_{t}^{(\beta)}$ represents the contribution to the volatility of the weather index independent from time and associated with the intercepts $\beta$ 's; $\left(R_{t}^{(S)}\right)_{t}$ is the idiosyncratic component of the volatility, and collects all the idiosyncratic random noises associated with the risks of each single DATs; $\left(R_{t}^{(\gamma)}\right)_{t}$ is associated with the common stochastic noise independent from the index $i$.

The long-term memory property of the time series $\left(\mathbf{R}_{t}\right)_{t}$ will be explored by implementing a two-step procedure:

- first, we detect the persistence property of any single component of $\left(\mathbf{R}_{t}\right)_{t}$

- second, we perform an aggregation of components to assess the value of the Hurst exponent of the entire series $\left(\mathbf{R}_{t}\right)_{t}$.

Remark 3.1. The long term memory is a property related to the time components of a series, even if it is generated by a stochastic process. Thus, it is evident that $\left(R_{t}^{(\beta)}\right)_{t}$ cannot concur in assessing the long term memory property of the process $\left(\mathbf{R}_{t}\right)_{t}$, and such a term does not appear in the single components analysis.

In the next theorem, we provide a theoretical estimate of the Hurst exponent associated with the component $\left(R_{t}^{(S)}\right)_{t}$, under suitable probabilistic conditions. To our purpose, we adopt the following:

Notation 3.2. We denote the beta distribution with parameters $a, b \in$ $(0,+\infty)$ by $B(a, b)$ distribution.

The density function of a $B(a, b)$ distribution is

$$
f(x)=\frac{1}{\beta(a, b)}(1-x)^{b-1} x^{a-1},
$$


where $\beta(a, b)$ is the normalizing constant.

Theorem 3.3. Let us assume that there exists $a \in(0,+\infty)$ and $b \in(0,2)$ such that $\eta_{i}$ are sampled by a $B(a, b)$ distribution, for each $i=1, \ldots, N$. Fixed $i=1, \ldots, N$, let $\left(S_{i, t}\right)_{t}$ be a stationary stochastic process such that

$$
\mathbb{E}\left[S_{i, t}\right]=0, \quad \forall i \in\{1, \ldots, N\}, t \in \mathbb{N}
$$

and

$$
\mathbb{E}\left[S_{i, u} S_{j, v}\right]= \begin{cases}\sigma_{S}^{2}, & \text { if } i=j \text { and } u=v \\ 0, & \text { otherwise. }\end{cases}
$$

Then, as $N \rightarrow+\infty$, the long-term memory property for $R_{t}^{(S)}$ holds, with Hurst exponent $H_{S}=1-b / 2$.

Proof. First of all, we need to show that

$$
\mathbb{E}\left[R_{t}^{(S)} R_{t-h}^{(S)}\right] \sim h^{-b}, \quad \text { as } N \rightarrow+\infty, h \rightarrow+\infty .
$$

Let us fix $t>0, h>0$ and examine $R_{t}^{(S)} R_{t-h}^{(S)}$.

$$
\begin{aligned}
& R_{t}^{(S)} R_{t-h}^{(S)}=\sum_{i=1}^{N} w_{i}^{2} \frac{1}{1-\eta_{i} L} S_{i, t} \sum_{j=1}^{N} w_{j}^{2} \frac{1}{1-\eta_{j} L} S_{j, t-h}= \\
& =\sum_{i=1}^{N} w_{i}^{2}\left[\sum_{l=0}^{\infty}\left(\eta_{i} L\right)^{l}\right] S_{i, t} \cdot \sum_{j=1}^{N} w_{j}^{2}\left[\sum_{m=0}^{\infty}\left(\eta_{j} L\right)^{m}\right] S_{j, t-h} .
\end{aligned}
$$

The terms of the series are positive, and so it is possible to exchange the order of the sums:

$$
R_{t}^{(S)} R_{t-h}^{(S)}=\sum_{m=0}^{\infty} \sum_{l=0}^{\infty} \sum_{i=1}^{N} \sum_{j=1}^{N} w_{i}^{2} w_{j}^{2} \eta_{i}^{l} \eta_{j}^{m} S_{i, t-l} S_{j, t-h-m} .
$$

In the limit as $N \rightarrow+\infty$ and setting $x:=\eta_{i}, y:=\eta_{j}$, (14) becomes:

$$
R_{t}^{(S)} R_{t-h}^{(S)} \sim \sum_{m=0}^{\infty} \sum_{l=0}^{\infty} \int_{0}^{1} \int_{0}^{1} x^{l} y^{m} S_{x, t-l} S_{y, t-h-m} \mathrm{~d} F(x, y),
$$

where $F$ is the joint distribution over $x$ and $y$.

By applying the expected value operator and by using the hypotheses on the $\eta$ 's and the $S$ 's, we get:

$$
\mathbb{E}\left[R_{t}^{(S)} R_{t-h}^{(S)}\right] \sim \frac{1}{\beta(a, b)} \sigma_{S}^{2} \sum_{m=0}^{\infty} \int_{0}^{1}(1-x)^{b-1} x^{2 m+h+a-1} \mathrm{~d} x=
$$




$$
\frac{1}{\beta(a, b)} \cdot \sigma_{S}^{2} \sum_{m=0}^{\infty} \frac{\Gamma(2 m+h+a) \Gamma(b)}{\Gamma(2 m+h+a+b)} .
$$

By Stirling formula, as $h \rightarrow+\infty$, we have that:

$$
\begin{gathered}
\frac{\Gamma(2 m+h+a)}{\Gamma(2 m+h+a+b)} \sim \\
\sim \frac{\sqrt{2 \pi(2 m+a+h+b)}}{\sqrt{2 \pi(2 m+a+h)}}\left(\frac{2 m+h+a}{e}\right)^{2 m+h+a}\left(\frac{2 m+h+a+b}{e}\right)^{-(2 m+h+a+b)} \sim \\
\sim \frac{(2 m+h+a)^{2 m+h+a}}{(2 m+h+a+b)^{2 m+h+a+b}}=\left(\frac{2 m+h+a}{2 m+h+a+b}\right)^{2 m+h+a} \cdot(2 m+h+a+b)^{-b}= \\
=\left(1-\frac{b}{2 m+h+a+b}\right)^{(2 m+h+a+b)-b} \cdot(2 m+h+a+b)^{-b} \sim e^{-b} \cdot 1 \cdot h^{-b} \quad(17)
\end{gathered}
$$

Hence, by (16) and (17), the rate of decay of the autocorrelation function related to $R_{t}^{(S)}$ is $-b$ as $h$ goes to infinity. By using Rangarajan and Ding (2000), we obtain that the component $R_{t}^{(S)}$ has long term memory with Hurst exponent $H_{S}=1-b / 2$.

Next result provides the estimation of the Hurst exponent for the component associated with the common random noise of the volatility of the temperature index.

Theorem 3.4. Assume that $\left\{\gamma_{t}\right\}_{t \geq 0}$ is a stochastic process with i.i.d. terms, such that $\mathbb{E}\left[\gamma_{t}\right]=0$ and $\operatorname{Var}\left[\gamma_{t}\right]=\sigma^{2}$, for each $t \geq 0$.

Moreover, let us assume that there exists $a \in(0,+\infty)$ and $b \in(1 / 2,3 / 2)$ such that $\eta_{i}$ are sampled by a $B(a, b)$ distribution, for each $i=1, \ldots, N$.

Then, for $N \rightarrow+\infty$, we have that $\left\{R_{t}^{(\gamma)}\right\}_{t \geq 0}$ has long term memory, with the Hurst exponent $H_{\gamma}=3 / 2-b$.

Proof. In the limit for $N \rightarrow \infty$ we have

$$
\begin{gathered}
R_{t}^{(\gamma)} \sim \lim _{N \rightarrow+\infty} \sum_{i=1}^{N} w_{i}^{2} \cdot \frac{1}{1-\eta_{i} L} \gamma_{t}= \\
=\int_{0}^{1} \frac{1}{1-\eta L} \gamma_{t} d F(\eta)=\int_{0}^{1} \sum_{k=0}^{\infty}(\eta L)^{k} \gamma_{t} d F(\eta)= \\
=\sum_{k=0}^{\infty} \int_{0}^{1} \eta \gamma_{t-k} d F(\eta)=\sum_{k=0}^{\infty}\left[\int_{0}^{1} \eta^{k} d F(\eta)\right] \gamma_{t-k}=: \sum_{k=0}^{\infty} a_{k} \gamma_{t-k},
\end{gathered}
$$

where

$$
a_{k}=\int_{0}^{1} \eta^{k} d F(\eta)=\mathbb{E}\left[\eta^{k}\right]
$$


By the distributional assumption on the $\eta$ 's, we have

$$
a_{k}=\frac{1}{\beta(a, b)} \int_{0}^{1}(1-x)^{b-1} x^{a+k-1} \mathrm{~d} x=\frac{\Gamma(a+k) \Gamma(b)}{\Gamma(a+k+b)} .
$$

By (17) and (19), we have

$$
a_{k} \sim k^{-b} \quad \text { as } k \rightarrow+\infty .
$$

Granger (1980) and (20) assure that $\left\{R_{t}^{(\gamma)}\right\}_{t \geq 0}$ is an integrated process of order $d=1-b$. Thus, since $b \in(1 / 2,3 / 2)$, one gets that $\left\{R_{t}^{(\gamma)}\right\}_{t \geq 0}$ has long term memory, with Hurst exponent given by $H_{\gamma}=3 / 2-b$.

We are now ready to assess explicitly the long term memory property of the series $\left\{\mathbf{R}_{t}\right\}_{t \geq 0}$. At this aim, we adapt to our specific context a result due to Granger (1980).

Theorem 3.5. Suppose that $\eta_{i}$ are sampled by a $B(a, b)$ distribution, with $a \in(0,+\infty)$ and $b \in(1 / 2,3 / 2)$, for each $i=1, \ldots, N$.

Then, for $N \rightarrow+\infty$, we have that $\left\{\mathbf{R}_{t}\right\}_{t \geq 0}$ has long memory with Hurst exponent $H$ given by

$$
H= \begin{cases}H_{\gamma}, & \text { for } b \in(1 / 2,1) \\ 1 / 2, & \text { for } b=1 \\ H_{S}, & \text { for } b \in(1,3 / 2)\end{cases}
$$

Proof. Since $\left\{R_{t}^{(S)}\right\}_{t \geq 0}$ and $\left\{R_{t}^{(\gamma)}\right\}_{t \geq 0}$ are independent processes, then we are in the position of using Granger (1980), so that

$$
H=\max \left\{H_{S}, H_{\gamma}\right\}
$$

By Theorems 3.3 and 3.4, a straightforward computation gives the thesis.

It is worth to note that the Hurst exponent depends on the distribution of the $\eta$ 's. Specifically, the selection of the parameter $b$ in the Beta distribution leads to different shapes for the density function of the $\eta$ 's. This evidence will be the focus of next section.

\section{Analysis of the results}

This section presents some comments on the results obtained in the previous section.

Theorem 3.5 states that the long term memory of the volatility of the index $A(t)$ can be obtained under the condition that $\eta_{i}$ follows a $B(a, b)$ distribution, where $a$ is a generic positive constant while $b \in(1 / 2,3 / 2)$, for each 
$i=1, \ldots, N$. Furthermore, such a Theorem states that the Hurst exponent, $H$, varies with the parameter $b$. Specifically, the long term memory of the process $\left(\mathbf{R}_{t}\right)_{t}$ is driven by the idiosyncratic term or the common term, on the basis of the value of $b$. It could also appear that the process is pure randomness, i.e. $H=1 / 2$, when $b=1$.

Formula (21) assures that $H \in(1 / 4,1 / 2)$ for $b \in(1,3 / 2)$, and $H \in(1 / 2,1)$ for $b \in(1 / 2,1)$. Furthermore, $H$ decreases with respect to $b$. The range of variation of $H$ explains that the process $\left(\mathbf{R}_{t}\right)_{t}$ is persistent for $b \in(1 / 2,1)$ and becomes antipersistent for $b \in(1,3 / 2)$. The degree of persistence reduces as $b$ increases.

Persistence and antipersistence are opposite concepts, but allow to make forecasts on the long run evolution of the volatility of the temperature index. In few words, persistence means that the history is repeating itself and, in the long run, the riskiness of the index follows the same tendency observed in past realizations. Differently, antipersistence is associated with a reversion of the behavior of the historical trajectory of volatility, and an opposite tendency should be expected. The case of $H=1 / 2$ is the one related to uncorrelated data.

The meaning of the parameter $b$ is highly relevant. Indeed, the hypotheses on the $\eta$ 's describe an autoregressive coefficient whose distribution captures different cases.

We show here three cases, by selecting three values of the parameter $a$ : $a=1 / 2,1,5$. The corner cases for $b$ are shown, so that $b=1 / 2,1,3 / 2$. The resulting cases are nine in number. The shapes of the resulting density functions of the Beta distribution $B(a, b)$ are illustrated in Figure 1 - panels $\mathrm{a}-\mathrm{j}$.

\section{INSERT FIGURE 1 ABOUT HERE}

Caption: Density functions of the Beta distribution $B(a, b)$ with different values of the parameters: $a=1 / 2,1,5 ; b=1 / 2,1,3 / 2$.

For each $i=1, \ldots, N$, the distribution of the $\eta_{i}$ provides information on how the $t$-th realization of the series $\left(\tilde{\varepsilon}_{i, t}^{2}\right)_{t}$ depends on the $t-1$-th one. A distribution concentrated around zero (one) is associated with a small (large) impact of the previous realization of the process for determining the current one.

The case $a=b=1$ (panel d) coincides with the uniform distribution. A symmetric density function appears also in the case of $a=b=1 / 2$ (panel h) - even if the extremes 0 and 1 are in this case more probable than the values in the center of the intervals. These two cases are associated with a fair distribution among the high and low values of the variation ranges of the Beta distributions, but in the second case the intermediate values are 
assumed with a low probability.

The cases $a=5, b=1, a=5, b=1 / 2$ and $a=1, b=1 / 2$ (panels a, b and e, respectively) exhibit a common behavior, with a rapid growth of the density function and high probabilities of taking values close to one. Such a tendency can also be appreciated when $a=5, b=3 / 2$ (panel c), even if in a less visible way. In this specific case, there is an internal global maximum of the density function in a point rather close to one.

The converse situation appears when $a=1, b=3 / 2$ and $a=1 / 2, b=3 / 2$ (panels $\mathrm{f}$ and $\mathrm{j}$, respectively), where we observe a high probability for values close to zero. However, such a behavior is more evident in the latter case, while in the former the density function follows a concave path.

The cases discussed above let us conjecture that low values for $a$ could be more likely associated with the antipersistence of the series when the $(t-1)$ th term plays a not so relevant role in identifying $\tilde{\varepsilon}_{i, t}^{2}$, while high values for $a$ are more likely associated with the persistence and high impact of $\tilde{\varepsilon}_{i, t-1}^{2}$ on $\tilde{\varepsilon}_{i, t}^{2}$. This is a nice result suggesting also future empirical developments.

\section{An analysis based on observed data}

In this Section, via a time series approach, we focus on the DAT dynamics of four cities located in the Eastern area of United States: Baltimore, Boston, Cincinnati and Philadelphia. As discussed in Section 2, truncated Fourier series have been included in both mean and volatility to capture seasonality. Furthermore, in the attempt of incorporating the stylized facts of temperature, autoregressive models for the mean and conditional variance have been also considered (see Equations 1-3). The DATs' dynamics of the aforementioned cities are calibrated on the historical series provided by the MET Office (UK), for the period going from January $1^{\text {st }}$, 1997 to January $31^{\text {st }}, 2006$. The time series have been converted from Fahrenheit to Celsius. As shown in Section 2, the seasonal mean for each DAT series $T_{i}(t)$ is modeled by equation (1). The seasonality function $F_{i, t}$ is written as a low-ordered Fourier series of period $2 \pi$ and integrable in the interval $[-\pi, \pi]$. This allows to decompose any periodic signal into the sum of a set of simple oscillating functions, producing a smooth progression of temperatures through seasons and making the model very parsimonious. In Table 1 the fitted parameters of the mean model, all significant at $5 \%$ level, for the seasonality functions, $F_{i, t}$, are presented. For each DAT, according to the Akaike and Schwartz Information criteria, all the truncation parameters $L_{i}$ were set equal to 8 . In Figure 1 the fitted Fourier series against raw data are shown.

INSERT TABLE 1 ABOUT HERE.

Caption Fitted parameters for the seasonality function, $F_{i, t}$. 
INSERT FIGURE 2 ABOUT HERE.

Caption DATs of Baltimore, Boston, Cincinnati and Philadephia together with the seasonal function from 1/1/1997 through 1/31/2006.

Apart from seasonality, the cyclical dynamics are calibrated by using an autoregressive process with $P$ lags, under the assumption of normality in the residuals, as in Equation (1). The models are calibrated on the deseasonalized DATs by using MLE. The selection of the most adequate $P$ is carried out by using both the Akaike and Schwartz information criteria.

Table 2. reports the regression parameters and, between brackets, the $t$ Statistics, for each of the four considered DAT time series.

\section{INSERT TABLE 2 ABOUT HERE}

Caption Fitted regression parameters of $A R(P)$ model.

The partial autocorrelation functions of the deseasonalized DAT time series are plotted in Figure 3, showing that AR(3) models for each of the four considered US time series are the most suitable ones.

\section{INSERT FIGURE 3 ABOUT HERE}

Caption Partial autocorrelation functions of the deseasonalized DAT time series.

By (3), we can estimate the conditional variances $\mathbb{E}\left(\epsilon_{i, t}^{2} \mid \mathcal{F}_{t-1}\right)$ in (5) through a MLE procedure for all the considered DATs. In Figure 4 the estimated conditional variances for all the DAT time series are shown.

\section{INSERT FIGURE 4 ABOUT HERE}

Caption Estimated Conditional Variance for each DAT series.

We computed the variance of the index as in Equation (5), equally weighting the estimated conditional volatility for each DAT time series. In order to perform the numerical estimates of the Hurst exponent $H$, we used the subroutine fastdfa.m (Little et al., 2006), which implements the Detrended Fluctuation Analysis (DFA). The problem of the error of the estimate of DFA and its dependence on the length of the time series is well known (Weron, 2002). Table 1 in (Weron, 2002) reports the standard deviation of time series with several lengths. We work with time series with length 3,652. The closest lengths examined in (Weron, 2002) are 2,048 and 4,096 , with standard deviations equal to 0.0355 and 0.0278 , respectively. To 
refine the estimate we calculated the confidence interval running the subroutine fastdfa 100,000 times on random time series with length 3,000, that is approximately the length of our sample. The standard deviations is 0.0306 , and $99 \%$ of the values fall in $(0.41,0.59)$.

In the empirical analysis, we follow a stepwise approach. First, we run the subroutine on each single estimated conditional variance and found the Hurst exponents equal to: 0.57 (Baltimora), 0.55 (Boston), 0.54 (Cincinnati), 0.59 (Philadelphia). Considering the error estimate, they all fall in the $H=0.5$ confidence interval.

Secondly, we computed the volatility of the index as in Equation (5) that is a equally weighted mixture of each conditional variance series. The resulting estimated Hurst exponent is $H=0.57$. Therefore, the empirical procedure allows to provide an estimation of the long term memory of the aggregated temperature index on the basis of its weighted components.

\section{Conclusions}

The paper considered the construction of a temperature index which can represent the underlying of a weather derivative. The main aim is to analyze the long term memory of the volatility of the proposed index. The usefulness of such an index lies, not only in its ability of controlling volume risk, but, for instance, also in providing an overall description of the temperatures in particular geographical areas. The analysis on the long term memory of the conditional volatility is based on the Hurst exponent and a key role is played by the Beta distribution of the coefficients, that has been selected because it can meet many different shapes. The distribution of the spatial coefficients influences the long term memory of the aggregate.

Indeed, the main theorem shows the range of values leading to short term memory, persistence, or antipersistence. Further developments of the present work include both theoretical and applied analysis.

From a theoretical point of view, models for the exploration of other different stylized facts of the temperature index may be considered. In this respect, for instance, the presence of regimes in the index dynamics can be explored. Such line of research could be quite relevant for commodities, since it can be foreseen a dependence of the long term behavior of the volatility of the index on the switching probabilities of each single component.

As it is shown by the application of the theoretical results on the observed data, the proposed approach may lead to scenario analysis based on the most feasible distribution of the coefficients, to the final aim of improving the estimate of risk through the long-run dependence. 


\section{Acknoweldgements}

The authors thank Dr. Ing. R. de Kok for fruitful management of data.

\section{References}

[1] Alexandridis A., Zapranis A.,D., 2013. Weather Derivatives: Modeling and Pricing Weather-Related Risk. Springer Science \& Business Media.

[2] Ausloos, M., Ivanova, K., 1999. Low q-moment multifractal analysis of gold price, Dow Jones Industrial Average and BGL-USD exchange rate. European Physical Journal B 8, 665-669.

[3] Ausloos, M., Ivanova, K., 2000. Introducing False EUR and False EUR exchange rates. Physica A 286, 353-366.

[4] Benth, F.E., 2003. On arbitrage-free pricing of weather derivatives based on fractional Brownian motion. Applied Mathematical Finance 10, 303-324.

[5] Benth, F.E., Saltyte Benth, J., 2013. Modeling and pricing in financial markets for weather derivatives, World Scientific.

[6] Bertrand J.-L., Parnaudeau M., 2017. Weather Threatens Businesses. It?s Time to Measure and Disclose the Risks, Harward Business School, https://hbr.org/2017/09/severe-weather-threatens-businessesits-time-to-measure-and-disclose-the-risks

[7] Bianchi, S., Pantanella, A., Pianese, A., 2013. Modeling stock prices by multifractional Brownian motion: an improved estimation of the pointwise regularity. Quantitative Finance 13, 1317-1330.

[8] Bloch, D., Annan, J., Bowles, J., 2011. Applying climate derivatives to flood risk management. Wilmott, 2011(56), 88-103.

[9] Bollerslev T., 1986. Generalized autoregressive conditional heteroskedasticity. Journal of Econometrics, 31, 307-327.

[10] Bollerslev, T., Mikkelsen, H.O., 1996. Modelling and pricing long memory in stock market volatility. Journal of Econometrics, 73, 151-184.

[11] Bouchaud, J.P., Gefen, Y., Potters, M., Wyart, M., 2004. Fluctuations and response in financial markets: The subtle nature of random price changes. Quantitative Finance, 4(2) 176-190.

[12] Brock, W.A., Hommes, C.H., 1997. Rational route to randomness. Econometrica 65, 1059-1095. 
[13] Brock, W.A., Hommes, C.H., 1998. Heterogeneous beliefs and routes to chaos in a simple asset pricing model. Journal of Economic Dynamics \& Control, 22, 1235-1274.

[14] Brody, C.D., Syroka, J., Zervos, M., 2002. Dynamical Pricing of Weather Derivatives. Quantitave Finance, 2, 189-198.

[15] Buckley N., Hamilton A., Harding J., Roche N., Ross N., Sands E., Skelding R., Watford N., Whitlow H., 2002. European weather derivatives. In General Insurance Convention.

[16] Bunn, D.W. and Oliveira, F.S., 2003. Evaluating individual market power in electricity markets via agent-based simulation. Annals of Operations Research 121(1-4), 57-77.

[17] Campbell S.D., Diebold F.X., 2005. Weather forecasting for weather derivatives. Journal of the American Statistical Association 100, 6-16.

[18] Cao M., Li A., and Wei J.Z., 2004. Watching the weather report. Canadian Investment Review 17, 27-33.

[19] Cao M., Wei J.Z., 2004. Weather derivatives valuation and market price of weather risk. Journal of Futures Markets 24, 1065-1089.

[20] Cerqueti, R., Rotundo, G., 2012. The Role of Diversity in Persistence Aggregation. International Journal of Intelligent Systems 27, 176-187.

[21] Cerqueti, R. and Rotundo, G., 2015. A review of aggregation techniques for agent-based models: understanding the presence of long-term memory. Quality \& Quantity 49, 1693-1717.

[22] Cheung, Y.W., Lai, K.S., 1993. A fractional cointegration analysis of purchasing power parity. Journal of Business and Economic Statistics 11, 103-112.

[23] Ding, Z., Granger, C.W.J., 1996a. Modelling volatility persistence of speculative returns: a new approach. Journal of Econometrics 73, 185200 .

[24] Ding, Z., Granger, C.W.J., 1996b. Varieties of long memory models. Journal of Econometrics 73, 61-77.

[25] Dorfleitner G., Wimmer M., 2010. The pricing of temperature futures at the Chicago Mercantile Exchange. Journal of Banking \& Finance, $34,1360-1370$.

[26] Eliazar, I., Klafter, J., 2005. On the nonlinear modeling of shot noise. Proceedings of the National Academy of Sciences of the United States of America, 102(39), 13779-13782. 
[27] Engle R.F., 1982. Autoregressive conditional heteroscedasticity with estimates of the variance of United Kingdom inflation. Econometrica $50,987-1007$.

[28] Foellmer, H., Horst, U., Kirman, A., 2005. Equilibria in financial markets with heterogeneous agents: a probabilistic perspective. Journal of Mathematical Economics 41, 123-155.

[29] Franses P.H., Neele J., van Dijk D., 2001. Modeling asymmetric volatility in weekly Dutch temperature data. Environmental Modelling \& Software 16, 131-137.

[30] Geman H., Leonardi M.P., 2005. Alternative approaches to weather derivatives pricing. Managerial Finance 31, 46-72.

[31] Golden, L. L., Wang, M., Yang, C., 2007. Handling weather related risks through the financial markets: Considerations of credit risk, basis risk, and hedging. Journal of Risk and Insurance, 74(2), 319-346.

[32] Granger, C.W.J., 1980. Long memory relationships and the aggregation of dynamic models. Journal of Econometrics 14, 227-228.

[33] Gulpinar, N., Canakoglu, E., 2017. Robust portfolio selection problem under temperature uncertainty, European Journal of Operational Research 256(2), 500-523.

[34] Hardle, W.K., Osipenko, M., 2011. Spatial risk premium on weather derivatives and hedging weather exposure in electricity. SFB 649 discussion paper.

[35] Hommes, C.H., 2001. Financial Markets as nonlinear adaptive evolutionary systems. Quantitative Finance 1, 149-167.

[36] Hurst, H., 1951. Long Term Storage Capacity of Reservoirs. Transactions of the American Society of Civil Engineers 116, 770-799.

[37] Hurst, H.E., 1957. A suggested statistical model of some time seris which occur in nature. Nature 180, 494.

[38] Ivanova, K., Ausloos, M., 1999. Low-order variability diagrams for short-range correlation evidence in financial data: BGL-USD exchange rate, Dow Jones industrial average, gold ounce price, Physica A 265, 279-291.

[39] Kirman, A.P., Teyssiere, G., 2002. Microeconomic models for longmemory in the volatility of financial time series. Studies in Nonlinear Dynamics and Econometrics 5, 281-302. 
[40] Lillo, F., Mike, S., Farmer, J.D., 2005. Theory for long memory in supply and demand. Physical Review E, 71(6), 066122.

[41] Little, M., McSharry, P., Moroz, I., Roberts, S., 2006. Nonlinear, biophysically-informed speech pathology detectionin 2006 IEEE International Conference on Acoustics, Speech and Signal Processing. ICASSP 2006 Proceedings.: Toulouse, France. pp. II-1080-II-1083.

[42] Lo, A.W., 1991. Long memory in stock market prices, Econometrica $59,1279-1313$.

[43] Lux, T., Ausloos, M., 2002. Market Fluctuations I: Scaling, Multiscaling and their Possible Origins. In: The Science of Disaster: Scaling Laws Governing Weather, Body, Stock-Market Dynamics", A.Bunde, J. Kropp, and H.-J.Schellnhuber, Eds. (Springer Verlag, Berlin) 377-413.

[44] Mandelbrot, B.B., Wallis, J., 1968. Noah, Joseph and operational hydrology. Water Resources Research 4, 909-918.

[45] Markovich, N.M. and Kilpi, J., 2009. Bivariate statistical analysis of TCP-flow sizes and durations. Annals of Operations Research 170(1), 199-216.

[46] McLeod, A.I., Hipel, A.W., 1978. Preservation of the rescaled adjusted range, 1: a reassessment of the Hurst phenomenon. Water Resources Research 14, 491-508.

[47] Müller A., Grandi M., 2000. Weather derivatives: a risk management tool for weather-sensitive industries. Geneva Papers on Risk and Insurance. Issues and Practice 25(2), 273-287.

[48] Rangarajan, G., Ding, M., 2000. Integrated approach to the assessment of long range correlation in time series data. Physical Review E 61(5), 4991-5001.

[49] Reboredo, J.C., Rivera-Castro, M.A., Miranda, J.G.V., García-Rubio, R., 2013. How fast do stock prices adjust to market efficiency? Evidence from a detrended fluctuation analysis. Physica A 392, 1631-1637.

[50] Saltyte Benth, J., Benth, F.E., Jalinskas, P., 2007. A Spatial-temporal Model for Temperature with Seasonal Variance. Journal of Applied Statistics, 34(7), 823-841.

[51] Saltyte Benth, J., Saltyte, L., 2011. Spatial-temporal model for wind speed in Lithuania. Journal of Applied Statistics, 38(6), 1151-1168.

[52] Schiller F., Seidler G., Wimmer M., 2012. Temperature models for pricing weather derivatives. Quantitative Finance 12, 489-500. 
[53] Smith, W., Harris, C.M., 1987. Fractionally differenced models for water quality time series. Annals of Operations Research 9(1), 399-420.

[54] Stulec, I., 2017. Effectiveness of weather derivatives as a risk management tool in food retail: The case of Croatia. International Journal of Financial Studies, 5(1), 2.

[55] Syroka, J.I., Toumi, R., 2001. Scaling and persistence in observed and modelled surface temperature. Geophys. Res. Lett. 28, 3255-3259.

[56] Taylor J.W., Buizza R., 2004. A comparison of temperature density forecasts from GARCH and atmospheric models. Journal of Forecasting $23,337-355$.

[57] Taylor J.W., Buizza R., 2006. Density forecasting for weather derivative pricing. International Journal of Forecasting 22, 29-42.

[58] Tsonis, A.A., Roebber, P.J., Elsner, J.B. 1999. Long-range correlations in the extratropical atmospheric circulation: origins and implications. J. Clim. 12, 1534-1541.

[59] Vandewalle, N., Ausloos, M., 1998. Spareness and Roughness of Foreign Exchange Rates. International Journal of Modern Physics C 9, 711-720.

[60] Wei, A., Leuthold, R.M., 2000. Agricultural Futures Prices and Long Memory Processes. OFOR Working Paper No. 00.04. Available at SSRN: http://ssrn.com/abstract $=229795$ or doi:10.2139/ssrn. 229795 .

[61] Weron, R., 2002. Estimating long-range dependence: finite sample properties and confidence intervals. Physica A: Statistical Mechanics and its Applications, 312(1):285-99.

[62] Yang, C.C., Li, L.S., Wen, M.M., 2011. Weather risk hedging in the European markets and international investment diversification. The Geneva Risk and Insurance Review, 36(1), 74-94.

[63] Zaffaroni, P., 2007. Memory and aggregation for models of changing volatility. Journal of Econometrics 136, 237-249.

[64] Zambrano, C. and Olaya, Y., 2016. An agent-based simulation approach to congestion management for the Colombian electricity market. Annals of Operations Research, doi:10.1007/s10479-016-2222-4.

[65] Zara, C., 2010. Weather derivatives in the wine industry. International Journal of Wine Business Research, 22(3), 222-237.

[66] Zhao, Y., Chang, S. and Liu, C., 2015. Multifractal theory with its applications in data management. Annals of Operations Research 234(1), 133-150. 